\title{
On propagation of boundary continuity of holomorphic functions of several variables
}

\author{
SALLA FrANZÉN AND BURGLIND JÖRICKE
}

\begin{abstract}
We prove that continuity properties of bounded analytic functions in bounded smoothly bounded pseudoconvex domains in two-dimensional affine space are determined by their behaviour near the Shilov boundary. Namely, if the function has continuous extension to an open subset of the boundary containing the Shilov boundary it extends continuously to the whole boundary. If it is e.g. Hölder continuous on such a boundary set, it is Hölder continuous on the closure of the domain. The statements may fail if the boundary is not smooth.
\end{abstract}

Mathematics Subject Classification (2000): 32A40 (primary); 32E35 (secondary).

\section{Introduction}

It is a classical observation that properties of analytic functions can be often directly read off from respective properties of their boundary values. The close relation between functions and boundary values has played a big role in complex analysis and its applications, for instance in operator theory modeled on function spaces, scattering theory and others. One side of this relation, the idea to study objects by considering canonical extensions, has been successfully applied in many other situations, as harmonic extension of functions, extension of elements of Kleinian groups to hyperbolic space or a respective recent construction in conformal geometry. One of the aspects of the relation of properties of analytic functions and their boundary values is comparison of the respective continuity properties.

Investigation of problems of this kind was initiated by a classical theorem of Hardy and Littlewood about contour and solid Hölder continuity. Recall that a function $f$ on a subset $E$ of $\mathbb{R}^{n}$ is Hölder continuous of order $\alpha \in(0,1]$ if there is a constant $C$ such that

$$
\sup _{\substack{z, z^{\prime} \in E \\\left|z-z^{\prime}\right|<\delta}}\left|f(z)-f\left(z^{\prime}\right)\right| \leq C \delta^{\alpha} \text { for all } \delta>0 .
$$

The smallest constant $C$ for which (1.1) holds is called the Hölder seminorm of $f$. 
For a domain $G$ in $\mathbb{C}^{n}(n \geq 1)$ we denote as usual by $A(G)$ the algebra of analytic functions in $G$ which extend continuously to the closure $\bar{G}$. The following result of Hardy and Littlewood concerns the case when $G$ equals $\mathbb{D}$, the unit disc in the complex plane [7].

Theorem HL. If $f \in A(\mathbb{D})$ and its restriction to the unit circle is Hölder continuous of order $\alpha, \alpha \in(0,1)$, then $f$ is Hölder continuous of the same order in the closed disc $\overline{\mathbb{D}}$.

It was proved later by Sewell [15] that actually the Hölder seminorms of the function on $\overline{\mathbb{D}}$, respectively on $\partial \mathbb{D}$, coincide.

In the sequel more general results on contour and solid continuity were proved. Results were obtained for very general domains in the complex plane and arbitrary quality of continuity instead of Hölder continuity. The quality of continuity is measured by the modulus of continuity. We call a continuous non-negative, nondecreasing function $\mu$ on the positive half-axis $[0,+\infty)$ a modulus of continuity if $\mu(0)=0$ and $\mu$ is subadditive, i.e.

$$
\mu\left(\delta_{1}+\delta_{2}\right) \leq \mu\left(\delta_{1}\right)+\mu\left(\delta_{2}\right) \text { for } \delta_{1} \geq 0, \delta_{2} \geq 0 .
$$

Note that for any continuous function $g$ on a compact convex set $E \subset \mathbb{R}^{n}$ the function $\mu_{g}(\delta)=\sup _{z, z^{\prime} \in E}\left|g(z)-g\left(z^{\prime}\right)\right|$ for $\delta>0, \mu_{g}(0)=0$, is a modulus of continuity. $\left|z-z^{\prime}\right|<\delta$

For any continuous function $f$ on an arbitrary compact subset $E$ of $\mathbb{R}^{n}$ there exists a modulus of continuity $\mu$ such that

$$
\sup _{\substack{z, z^{\prime} \in E \\\left|z-z^{\prime}\right|<\delta}}\left|f(z)-f\left(z^{\prime}\right)\right| \leq \mu(\delta) \text { for all } \delta>0 .
$$

Indeed, it is enough to extend $f$ to a continuous function on a large closed ball and apply the preceding argument.

For unifying statements it will be often more convenient to use the following more general notion instead. Call a continuous non-negative, non-decreasing function $\mu$ on the positive half-axis $[0,+\infty)$ a generalized modulus of continuity if

$$
\mu(n \delta) \leq n \mu(\delta)
$$

for all natural numbers $n$ and $\delta>0$. We do not require here that $\mu(0)=0$. If

$$
\sup _{\substack{z, z^{\prime} \in E \\\left|z-z^{\prime}\right|<\delta}}\left|f(z)-f\left(z^{\prime}\right)\right| \leq C \mu(\delta) \text { for all } \delta>0
$$

for a generalized modulus of continuity $\mu$, then we call $f \mu$-continuous on $E$.

The more general point of view allowed to understand the underlying mechanism. It has a local and a global aspect. The local aspect is of potential theoretic 
nature and based on the existence of local barrier functions. Local results hold for domains which are at a given point fat enough in a potential theoretic sense. The global aspect is based on the maximum principle for analytic functions.

The problem becomes more subtle for analytic functions of several variables. Moreover, natural questions arise which do not exist in dimension one. Already the afore mentioned global aspect is more subtle for analytic functions in multidimensional domains. Indeed, for a bounded domain $G \subset \mathbb{C}^{n}, n>1$, any function in $A(G)$ attains its maximum on the Shilov boundary $S(G)$ of the domain (The definition of Shilov boundary will be recalled below). Notice that $S(G)$ may be considerably smaller than the boundary $\partial G$ of the domain. The question arises whether the continuity properties of functions in $A(G)$ can be read off from those of their restrictions to a much smaller set than the boundary of the domain.

Moreover, the question arises whether one can weaken the a priori requirement $f \in A(G)$. Will an analytic function in $G$ be automatically in $A(G)$ if it extends continuously to a smaller subset of the boundary? This question has no analogue in dimension 1. Here are the precise questions which emerge from a paper of Glicksberg [5] although they are not asked there explicitly.

Question 1.1. For which bounded domains $G \subset \mathbb{C}^{n}$ any continuous function on $G \cup S(G)$ that is bounded and analytic on $G$ extends to a continuous function on the closure $\bar{G}$ ?

Question 1.2. For which bounded domains $G \subset \mathbb{C}^{n}$ the previous question has an affirmative answer if $S(G)$ is replaced by any relatively open subset $V$ of $\partial G$ with $V \supset S(G)$ ?

Glicksberg himself gave sufficient conditions on a domain to ensure a positive answer to the Question 1.1. Unfortunately, the conditions are difficult to verify and are only sufficient, which motivates the search for more geometric criteria. For a statement of his theorem we refer to the original paper [5].

In [9] it is proved that for regular Weil polyhedra in $\mathbb{C}^{n}, n>1$, Question 1.1 has a positive answer. Glicksberg's conditions may not be satisfied for regular Weil polyhedra. Weil polyhedra are defined in the following way.

Let $f_{j}, j=1, \ldots, N$ be analytic functions in an open subset $U$ of $\mathbb{C}^{n}$. Suppose the set $G \stackrel{\text { def }}{=}\left\{z \in U:\left|f_{j}(z)\right|<1, j=1, \ldots, N\right\}$ is connected and relatively compact in $U$. Then $G$ is called a Weil polyhedron. Weil polyhedra are pseudoconvex domains. The polyhedron is regular if the level sets $\left\{\left|f_{j}\right|=1\right\}$ of any $k$-tuple $(k \leq n)$ of different functions $f_{j}$ intersect in general position. Note that polydiscs are regular Weil polyhedra. Moreover, all polynomially convex sets can be approximated from the outside by regular Weil polyhedra. The Shilov boundary of regular Weil polyhedra has a simple description (see [2]). It has real dimension $n$.

In [9] it is also shown that for $G$ being any regular Weil polyhedron and $\mu$ being any generalized modulus of continuity, $\mu$-continuity on $S(G)$ for a function as in Question 1.1 implies $\mu$-continuity of the function on the whole closure $\bar{G}$ of $G$.

On the other hand Glicksberg showed that for the "Hartogs triangle" $G \stackrel{\text { def }}{=}$ $\left\{\left(z_{1}, z_{2}\right) \in \mathbb{C}^{2}:\left|z_{1}\right|<\left|z_{2}\right|<1\right\} \subset \mathbb{C}^{2}$ even the answer to Question 1.2 is negative. The Hartogs triangle does not have smooth boundary. It turns out that for 
domains in $\mathbb{C}^{2}$ this is the only obstruction for a positive answer to Question 1.2. We prove here the following theorem. The formulation was proposed as a conjecture in [10].

Theorem 1.3. Let $G \subset \mathbb{C}^{2}$ be a bounded pseudoconvex domain with $\mathcal{C}^{\infty}$ boundary. Let $V \subset \partial G$ be relatively open and contain the Shilov boundary $S(G)$. Then for any continuous function $f$ on $G \cup V$ that is bounded and analytic in $G$ the following two statements hold.

1. $f$ extends to a continuous function on the closure $\bar{G}$.

2. If $f$ is $\mu$-continuous on $V$ for a generalized modulus of continuity $\mu$ then $f$ is $\mu$-continuous on $\bar{G}$. The respective $\mu$-seminorms are related by a multiplicative constant depending only on $G$ and $V$.

It follows that if in addition $f$ is of class $\mathcal{C}^{\infty}$ on $G \cup V$ then $f$ extends to a $\mathcal{C}^{\infty}$ function on the closure $\bar{G}$.

We do not know whether in Theorem 1.3 one can replace the neighbourhood $V$ of the Shilov boundary by the Shilov boundary itself. We do not know either in which way the latter would be related to the still open problem whether biholomorphic mappings between bounded smoothly bounded pseudoconvex domains have smooth extension to the boundary.

ACKNOwledgements. The present paper was written while the second author was a guest of the Max-Planck-Institute.

\section{Proof of the theorem}

We start with recalling some known results and notions. The first two statements extract the local and the global aspect of the relation of solid and boundary continuity of analytic functions. They hold in arbitrary dimensions. We refrain from formulating the more subtle results that are known in dimension one and refer to the original literature (see e.g. [14]).

We start with the following global statement.

Lemma I. Let $G$ be a bounded domain in $\mathbb{C}^{n}$ and let $f \in A(G)$. Then for every $\delta>0$ the supremum

$$
\sup \left\{\left|f(z)-f\left(z^{\prime}\right)\right|: z, z^{\prime} \in \bar{G},\left|z-z^{\prime}\right|<\delta\right\}
$$

is attained when one of the points $z$ or $z^{\prime}$ is contained in the boundary.

The lemma is well-known (see for instance [14]). For convenience of the reader we present the short proof.

Proof of the Lemma. Denote $z^{\prime}=z+h$. Both points $z$ and $z+h$ are contained in $\mathrm{G}$ iff $z \in G \bigcap(G-h)$. Fix the complex vector $h$ and apply the maximum principle to the analytic function $z \rightarrow f(z+h)-f(z)$ in $G \bigcap(G-h)$. Since the boundary of the set $G \bigcap(G-h)$ is contained in the union of the boundaries $\partial G$ and $\partial(G-h)$, the supremum $\sup \{|f(z+h)-f(z)|: z \in G \bigcap(G-h)\}$ is attained if either $z \in \partial G$ or $z \in \partial(G-h)$. The second inclusion is equivalent to $z^{\prime}=z+h \in \partial G$. 
Here is a version of the local statement suitable also in the multi-dimensional setting. In the multi-dimensional setting it can be obtained from one-dimensional results by slicing. For an outline of the proof see [9].

Theorem I. Let $G$ be a domain in $\mathbb{C}^{n}$ with $z \in \partial G$. Assume that there is an open frustrum of a cone $K$ with vertex at $z$ lying in $\mathbb{C}^{n} \backslash \bar{G}$. Let $\mu$ be a generalized modulus of continuity. Then there exists a constant $C>0$ depending only on the aperture and height of the frustrum of the cone, such that for any function $f \in A(G)$ the relation

$$
\sup _{\substack{z^{\prime} \in \partial G \\\left|z-z^{\prime}\right|<\delta}}\left|f(z)-f\left(z^{\prime}\right)\right| \leq \mu(\delta) \text { for any } \delta>0
$$

implies the relation

$$
\sup _{\substack{z^{\prime} \in \bar{G} \\\left|z-z^{\prime}\right|<\delta}}\left|f(z)-f\left(z^{\prime}\right)\right| \leq C \mu(\delta) \text { for any } \delta>0 .
$$

The lemma and the theorem imply the statement on the relation between solid and boundary continuity for a wide class of multi-dimensional domains, in particular for those with $\mathcal{C}^{2}$ boundary. Indeed, if a domain has $\mathcal{C}^{2}$ boundary then for each of its boundary points the conditions of theorem I are satisfied and, moreover, the aperture and the height of the frustrum of the cones can be chosen to be independent of the boundary point.

Recall that the notion of the Shilov boundary was introduced in the general frame of Banach algebras. Here we consider only the case of the algebra $A(G)$ for an arbitrary domain $G \subset \mathbb{C}^{n}$. In this case the Shilov boundary $S(G)$ is the intersection of all closed subsets $S$ of $\bar{G}$ for which

$$
|f(z)| \leq \max _{S}|f| \text { for all } z \in \bar{G} \text { and for all } f \in A(G) .
$$

A set $S$ with the described property is called a boundary for $A(G)$. Note that $\partial G$ is a boundary, hence $S(G) \subset \partial G$. Moreover $S(G)$ is a boundary itself, i.e.

$$
|f(z)| \leq \max _{S(G)}|f| \text { for all } z \in \bar{G} \text { and for all } f \in A(G) .
$$

For more detailed information we refer to the book [4].

Bremermann [2] initiated research on a geometric characterization of the Shilov boundary. He conjectured that the Shilov boundary $S(G)$ of a pseudoconvex domain $G$ in $\mathbb{C}^{n}$ with boundary of class $\mathcal{C}^{2}$ is equal to the closure of the set $\Psi_{+}(G)$ of strictly pseudoconvex boundary points. The first theorem in this direction was proved by Rossi.

Theorem II. [13] If $G$ is a bounded pseudoconvex domain in $\mathbb{C}^{n}$ with $\mathcal{C}^{2}$ boundary and $\bar{G}$ admits a Stein neighbourhood basis, then

$$
S(G)=\overline{\Psi_{+}(G)} .
$$


For one of the inclusions it is enough to require that the domain has $\mathcal{C}^{2}$ boundary.

Theorem III. [1] Let $G$ be a bounded domain in $\mathbb{C}^{n}$ with $\mathcal{C}^{2}$ boundary. Then the Shilov boundary is contained in the closure of the set of strictly pseudoconvex boundary points, i.e.

$$
S(G) \subset \overline{\Psi_{+}(G)} .
$$

Note that the domain $G$ is not required to be pseudoconvex.

As for the other inclusion the condition of existence of a Stein neighbourhood basis of $\bar{G}$ can be removed, provided the boundary is of class $\mathcal{C}^{\infty}$ instead of $\mathcal{C}^{2}$.

Theorem IV. [12] Let $G \subset \mathbb{C}^{n}$ be a bounded pseudoconvex domain with $\mathcal{C}^{\infty}$ boundary. Then

$$
\overline{\Psi_{+}(G)} \subset S(G) .
$$

Using the geometric notion of strict pseudoconvexity of boundary points instead of the notion of the Shilov boundary one can give a statement that holds for arbitrary bounded domains in $\mathbb{C}^{2}$ with $\mathcal{C}^{3}$ boundary. Denote by $\Psi_{-}(G)$ the set of strictly pseudoconcave boundary points. Note that the set $\partial G \backslash\left(\overline{\Psi_{+}(G)} \cup \overline{\Psi_{-}(G)}\right)$ is foliated into one-dimensional complex manifolds.

The following theorem holds.

Theorem 2.1. Let $G \subset \mathbb{C}^{2}$ be a bounded domain in $\mathbb{C}^{2}$ with $\mathcal{C}^{3}$ boundary. Suppose $V_{+} \subset \partial G$ is relatively open in $\partial G$ and contains $\overline{\Psi_{+}(G)}$. Let $f$ be any continuous function on $G \cup V_{+}$which is bounded and analytic on $G$. Then

1. $f$ extends to a continuous function on $\bar{G}$.

2. If $f$ is $\mu$-continuous on $G \cup V_{+}$for a generalized modulus of continuity $\mu$ then it is $\mu$-continuous on $\bar{G}$. The $\mu$-seminorms on $G \cup V_{+}$and on $\bar{G}$ differ by a multiplicative constant depending on $G, V_{+}$and the supremum norm of $f$ on $G$.

In general, for non-pseudoconvex domains $G$ the Shilov boundary may be strictly

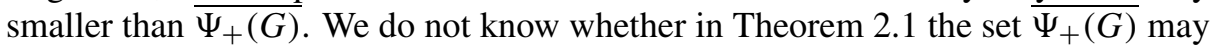
be replaced by the Shilov boundary itself.

The following lemmas relate Theorem 2.1 to Theorem 1.3.

Lemma 2.2. Let $G$ be as in Theorem 2.1 and $p \in \overline{\Psi_{-}(G)} \backslash \overline{\Psi_{+}(G)}$. Then there is a neighbourhood $U_{p}$ of $p$ in $\mathbb{C}^{2}$ which depends only on $G$ and $p$ such that each bounded analytic function in $G$ extends to an analytic function in $U_{p}$. The extension does not increase the supremum norm of the function.

Lemma 2.3. There exists a neighbourhood $V$ of $\Psi(G) \stackrel{\text { def }}{=} \Psi_{+}(G) \cup \overline{\Psi_{-}(G)}$ in $\partial G$ such that each function $f$ as in Theorem 2.1 has continuous extension to $G \cup V$. Moreover, $f$ is $\mu$-continuous on $V$ with $\mu$-seminorm differing from that on $V_{+}$by a multiplicative constant depending on $G, V$ and the supremum norm of $f$ on $G$.

Proof. Indeed, choose for each $U_{p}$ as in Lemma 2.2 two open subsets $V_{p}^{\prime}$ and $V_{p}^{\prime \prime}$ of $\partial G, p \in V_{p}^{\prime} \Subset V_{p}^{\prime \prime} \Subset U_{p} \cap \partial G$. Let $\stackrel{\circ}{V}_{+}$be an open subset of $\partial G, \overline{\Psi_{+}(G)} \subset \stackrel{\circ}{V}_{+} \Subset$ 
$V_{+}$. Cover the compact set $\overline{\Psi_{-}(G)} \backslash \stackrel{\circ}{V}_{+}$by a finite number of $V_{p_{j}}^{\prime}, j=1 \ldots, N$. Denote by $V$ the union $V=\stackrel{\circ}{V}_{+} \cup \bigcup_{j=1}^{N} V_{p_{j}}^{\prime}$. On each $V_{p_{j}}^{\prime \prime}$ the extension of $f$ is Lipschitz continuous with Lipschitz seminorm depending on the supremum norm of $f$ and the distance of $V_{p_{j}}^{\prime \prime}$ to the complement of $U_{p}$. Since any generalized modulus of continuity exceeds const $\cdot \delta, \delta \geq 0$, for some positive constant const, $f$ is $\mu$-continuous on each $V_{p_{j}}^{\prime \prime}$. It is also $\mu$-continuous on $V_{+}$and $\stackrel{\circ}{V_{+}} \Subset V_{+}$. Notice, that for small $\delta>0$ two points $z$ and $z^{\prime}$ in $V \subset \partial G$ of distance less than $\delta$ from each other belong both to one of the sets $V_{p_{j}}^{\prime \prime}$ or to $V_{+}$. It follows that $f$ is $\mu$-continuous on $V$ with $\mu$-seminorm depending on $G, V_{+}$and the supremum norm of $f$ on $G$.

Proof of Lemma 2.2. By the conditions of the lemma, $p$ is a minimal point of $\partial G$ in the sense that $\partial G$ contains no analytic curve that passes through $p$. For a small ball $B_{p}$ around $p$ the neighbourhood $W_{p}=\partial G \cap B_{p}$ of $p$ on $\partial G$ is pseudoconcave from the side of $G$. Denote the pseudoconvex set $B_{p} \backslash \bar{G}$ by $\Omega_{p}$. Let $W_{p}^{\prime} \Subset W_{p}$ be a smaller neighbourhood of $p$ on $\partial G$. By a theorem of Trepreau [16] (see also the more general result of Tumanov [17]) analytic functions on a neighbourhood of $W_{p}^{\prime}$ have analytic extension to a one-sided neighbourhood $\mathcal{O}_{p}$ of $p$ (i.e. to one of the connected components of $b_{p} \backslash \partial G$ for a small ball $b_{p}$ around $p$ ). The one-sided neighbourhood $\mathcal{O}_{p}$ depends only on $W_{p}^{\prime}$ and the extension preserves the supremum norm of the function. The set $\mathcal{O}_{p}$ is contained in $\Omega_{p}$. Indeed, take a vector $v$ so that for all sufficiently small positive numbers $\varepsilon$ the translates $W_{p}^{\prime}+\varepsilon v$ are contained in $\Omega_{p}$. Analytic functions in $\Omega_{p}$ extend to $\mathcal{O}_{p}+\varepsilon v$ for all small $\varepsilon>0$, hence $\mathcal{O}_{p}+\varepsilon v \subset \Omega_{p}$ for all such $\varepsilon$. It follows that $\mathcal{O}_{p} \subset \Omega_{p}$, and therefore $G \cup \partial G \cup \mathcal{O}_{p}$ covers the ball $b_{p}$. Applying Trepreau's theorem to a small translate $W_{p}^{\prime}-\varepsilon v$ into $G$, we obtain the lemma.

Proof of Theorems 1.3 and 2.1. To unify notation, put $\Psi(G)=\Psi_{+}(G) \cup \Psi_{-}(G)$ in both theorems (in case of Theorem $1.3 \Psi_{-}(G)$ is empty). We may assume that there is a neighbourhood $V$ of $\overline{\Psi(G)}$ in $\partial G$ such that $f$ is bounded and analytic in $G$, continuous in $G \cup V$ and $\mu$-continuous on $V$ for some generalized modulus of continuity $\mu$. (For Theorem 2.1 the present generalized modulus of continuity differs from the one in the statement of Theorem 2.1 by a multiplicative constant that depends on $G, V$ and the supremum norm of $f$ ). Choose a neighbourhood $\stackrel{\circ}{V}$ (in $\partial G$ ) of $\Psi(G), \stackrel{\circ}{V} \Subset V$.

The following lemma is a local statement. It implies immediately continuous extension of the function $f$ to the set $\partial G \backslash \stackrel{\circ}{V}$. Successive application of the lemma allows to increase the quality of continuity of the function on this set (see Corollary 2.5).

Lemma 2.4. Let $G, \stackrel{\circ}{V}$ and $V$ be as above. For each point $p \in \partial G \stackrel{\bar{\circ}}{V}$ there are a small ball $b_{p}$ around $p$, a positive number $\tau_{p} \leq 1$ and a constant $C_{p}>1$ such that the following holds. 
Suppose $f$ and $\mu$ are as above and $v \geq \mu$ is another generalized modulus of continuity. Suppose

$$
\sup _{\substack{z, z^{\prime} \in G \\\left|z-z^{\prime}\right|<\delta}}\left|f(z)-f\left(z^{\prime}\right)\right| \leq v(\delta) \text { for all } \delta>0 .
$$

Then

$$
\sup _{\substack{z, z^{\prime} \in b_{p} \cap G \\\left|z-z^{\prime}\right|<\delta}}\left|f(z)-f\left(z^{\prime}\right)\right| \leq C_{p} \nu(\delta)^{1-\tau_{p}} \mu(\delta)^{\tau_{p}} \text { for all } \delta>0 .
$$

The constants $\tau_{p}$ and $C_{p}$ depend only on $p, G, \stackrel{\circ}{V}$ and $V$ but not on $v$ and $\mu$.

Note that any function as in the beginning of the proof satisfies (2.1) with $\nu(\delta) \equiv c \sup _{G}|f|$ for any constant $c \geq 2$. Redefining $\mu(\delta)$ to be constant for $\delta>\operatorname{diam}(\bar{G})$ the constant $c$ may be chosen so that $\nu \geq \mu$.

We postpone the proof of the lemma. The lemma has the following corollary.

Corollary 2.5. Suppose $f$ is as above and satisfies (2.1) for a function $v$ as in the lemma. Then $f$ has continuous extension to $\partial G$. Moreover, there exist a positive constant $\tau \leq 1$ and a constant $C>1$ (depending only on $G$ and $V$ ) such that for all positive $\delta$

$$
\sup _{z, z^{\prime} \in \bar{G},\left|z-z^{\prime}\right|<\delta}\left|f(z)-f\left(z^{\prime}\right)\right| \leq C \nu(\delta)^{1-\tau} \mu(\delta)^{\tau}
$$

Proof of the Corollary. Inequality (2.2) implies that for each $p \in \partial G \stackrel{\circ}{V}$ the function $f$ extends continuously to $b_{p} \cap \bar{G}$, being uniformly continuous on the dense open subset $b_{p} \cap G$. Since $f$ extends continuously to $V \supset \stackrel{\circ}{V}$, continuous extension to the whole boundary $\partial G$ follows.

Put $V_{p} \stackrel{\text { def }}{=} b_{p} \cap \partial G$ and choose an open subset $\stackrel{\circ}{V}_{p}$ of $\partial G, p \in \stackrel{\circ}{V_{p}} \Subset V_{p}$. Cover the compact set $\partial G \backslash \stackrel{\circ}{V}$ by finitely many of the $\stackrel{\circ}{V_{p}}$. Let $\tau$ be the smallest of the respective constants $\tau_{p}$. Notice, that for small $\delta>0$ two points $z$ and $z^{\prime}$ in $\partial G$ belong both to one of the sets $V_{p}$ or to $V$ if $\left|z-z^{\prime}\right|<\delta$. Since $\mu(\delta) \leq \nu(\delta)$ for $\delta \geq 0$, and $\tau \leq \tau_{p}$, the inequality

$$
\sup _{\substack{z, z^{\prime} \in \partial G \\\left|z-z^{\prime}\right|<\delta}}\left|f(z)-f\left(z^{\prime}\right)\right| \leq \max C_{p} \cdot \nu(\delta)^{1-\tau} \mu(\delta)^{\tau}
$$

holds for small $\delta>0$. Increasing the constant, we get (2.4) for arbitrary $\delta>0$. Theorem I and Lemma I imply (2.3) after further increasing the multiplicative constant. The corollary is proved.

We will now finish the proof of the theorems by successively applying the corollary. 
End of proof of the theorems. Let $f$ be as in the beginning of the proof of the theorems. Put $\mu_{0}(\delta) \equiv A$ for $\delta \geq 0$, where $A=c \sup _{G}|f|$ for a suitable constant $c$ exceeding 2 so that $\mu_{0} \geq \mu$. Let $C$ and $\tau$ be the constants of the corollary. Applying the corollary with $v=\mu_{0}$ we obtain that $f$ extends to a continuous function in $\bar{G}$ and

$$
\sup _{\substack{z, z^{\prime} \in \bar{G} \\\left|z-z^{\prime}\right|<\delta}}\left|f(z)-f\left(z^{\prime}\right)\right| \leq C A^{1-\tau} \mu(\delta)^{\tau} \text { for all } \delta>0 .
$$

Put $\mu_{1}(\delta)=C A^{1-\tau} \mu(\delta)^{\tau}=C \mu_{0}(\delta)^{1-\tau} \mu(\delta)^{\tau}$ for $\delta \geq 0$. Then $\mu_{1}$ is non-negative, non-decreasing, satisfies condition (1.3) and is not smaller than $\mu$. We may apply the corollary with $v=\mu_{1}$. Repeated application of the corollary gives for any natural number $j$ the following estimate

$$
\sup _{\substack{z, z^{\prime} \in \bar{G} \\\left|z-z^{\prime}\right|<\delta}}\left|f(z)-f\left(z^{\prime}\right)\right| \leq \mu_{j}(\delta) \text { for all } \delta>0,
$$

where

$$
\mu_{j}(\delta)=C^{\sum_{l=0}^{j-1}(1-\tau)^{l}} A^{(1-\tau)^{j}} \mu(\delta)^{\tau \sum_{l=0}^{j-1}(1-\tau)^{l}}, \delta>0 .
$$

Indeed, suppose (2.5) is true for $j-1$ with the non-negative, non-decreasing function $\mu_{j-1}$ defined by (2.6). Suppose that $\mu_{j-1} \geq \mu$ and $\mu_{j-1}$ satisfies (1.3). Then the corollary gives

$$
\sup _{\substack{z, z^{\prime} \in \bar{G} \\\left|z-z^{\prime}\right|<\delta}}\left|f(z)-f\left(z^{\prime}\right)\right| \leq C\left(\mu_{j-1}(\delta)\right)^{1-\tau} \mu(\delta)^{\tau} \text { for all } \delta>0 .
$$

Denote the function on the right hand side by $\mu_{j}$. It is again non-negative, nondecreasing, not smaller than $\mu$ and satisfies (1.3), since both functions $\mu_{j-1}$ and $\mu$ have this property. Moreover, (2.6) holds for $\mu_{j}$. Indeed, since (2.6) is true for $j-1$,

$$
\begin{aligned}
\mu_{j}(\delta) & =C\left(C^{\sum_{l=0}^{j-2}(1-\tau)^{l}} A^{(1-\tau)^{j-1}} \mu(\delta)^{\tau \sum_{l=0}^{j-2}(1-\tau)^{l}}\right)^{1-\tau} \mu(\delta)^{\tau} \\
& =C^{\sum_{l=0}^{j-1}(1-\tau)^{l}} A^{(1-\tau)^{j}} \mu(\delta)^{\tau \sum_{l=0}^{j-1}(1-\tau)^{l}} \text { for } \delta \geq 0,
\end{aligned}
$$

what we needed to prove.

Note that

$$
\lim _{j \rightarrow \infty} \mu_{j}(\delta)=C^{\frac{1}{1-(1-\tau)}} \mu(\delta)^{\tau} \frac{1}{1-(1-\tau)}=C^{\frac{1}{\tau}} \mu(\delta) \text { for } \delta \geq 0
$$

Since (2.5) is true for each $j$, we obtain the theorem with constant $C^{\frac{1}{\tau}}$. Here $C$ and $\tau$ are the constants in the corollary. The theorems are proved.

It remains to prove the lemma. 
Proof of Lemma 2.4. Let $W$ be a connected component of $\partial G \backslash \overline{\Psi(G)}$. $W$ is a Leviflat hypersurface and hence it is foliated by 1-dimensional complex manifolds which are embedded or injectively immersed into $W$. Recall that $\stackrel{\circ}{V}$ is an open subset of $\partial G, \overline{\Psi(G)} \subset \stackrel{\circ}{V} \subset \stackrel{\circ}{V} \subset V$.

Take a point $p \in W \backslash \stackrel{\circ}{V}$. Let $\mathcal{L}_{p}$ be the Levi-leaf of $W$ through $p$.

There is a curve $\gamma_{p}$ in $\mathcal{L}_{p}, \gamma_{p}:[0,1] \rightarrow \mathcal{L}_{p}$, which joins $p$ with a point in $W \cap \stackrel{\circ}{V}$. Otherwise $\mathcal{L}_{p}$ would be contained in the compact subset $W \backslash \stackrel{\circ}{V}$ of $W$ and the same would apply to its closure $\overline{\mathcal{L}}_{p}$ (closure with respect to Euclidean topology in $\mathbb{C}^{2}$ ). As a consequence, $\overline{\mathcal{L}}_{p}$ would be a compact set which is the union of injectively immersed complex one-dimensional analytic manifolds (see also [11]), which is impossible [6]. Removing parts of $\gamma_{p}$ between self-intersection points and approximating, we may assume that $\gamma_{p}$ is real analytic on a slightly larger interval, in particular it extends to an analytic diffeomorphism from a neighbourhood of $I_{\varepsilon}=[-\varepsilon, 1]$ in $\mathbb{C}$ to a neighbourhood of $\gamma_{p}\left(I_{\varepsilon}\right)$ on $\mathcal{L}_{p}$. We may keep the condition $\gamma_{p}(0)=p \cdot \gamma_{p}\left(I_{\varepsilon}\right)$ can be considered as the symmetry axis of a rectangle in the complex leaf $\mathcal{L}_{p}$. Our goal is now to foliate a neighbourhood of $\gamma_{p}\left(I_{\varepsilon}\right)$ in $\bar{G}$ by holomorphic rectangles close to the mentioned one. More precisely, we will define a diffeomorphic map $\mathcal{H}\left(=\mathcal{H}_{p}\right)^{1}$ of class $\mathcal{C}^{1}$ from a neighbourhood of a closed rectangular box

$$
Q\left(=Q_{\varepsilon, \sigma}\right)=\left\{\left(z_{1}, z_{2}\right): \operatorname{Re} z_{1} \in I_{\varepsilon}, \operatorname{Im} z_{1} \in[-\varepsilon, \varepsilon], \operatorname{Re} z_{2} \in[-\sigma, \sigma], \operatorname{Im} z_{2} \in[0, \sigma]\right\}
$$

onto a subset of $\bar{G}$ satisfying the following properties:

(1) Denote by $R_{z_{2}}, z_{2} \in[-\sigma, \sigma] \times i[0, \sigma]$, the rectangle obtained from $Q$ by fixing $z_{2}$. The mapping $\mathcal{H}$ is holomorphic on each $R_{z_{2}}$.

(2) The "lower" face of $\mathcal{H}(Q), \mathcal{H}\left(Q \cap\left\{\operatorname{Im} z_{2}=0\right\}\right)$, is contained in $W \subset \partial G$. Each conformal rectangle $\mathcal{H}\left(R_{x_{2}}\right), x_{2} \in[-\sigma, \sigma]$, is contained in one of the leaves of $W$. In particular, $\mathcal{H}\left(R_{0}\right)$ contains $\gamma\left(I_{\varepsilon}\right)$.

This is obtained in the following way. Let $X$ and $Y$ be real $\mathcal{C}^{2}$ vector fields defined at points of $\mathcal{L}_{p}$ close to $\gamma\left(I_{\varepsilon}\right)$. Let $\mathcal{F}_{Y, y}, \mathcal{F}_{X, x}$ be their flows. We may choose the vector fields so that $\mathcal{H}(x+i y, 0) \stackrel{\text { def }}{=} \mathcal{F}_{Y, y} \circ \mathcal{F}_{X, x}(p)$ coincides with the analytic extension of $\gamma_{p}$ for $x, y$ in a neighbourhood of $I_{\varepsilon} \times[-\varepsilon, \varepsilon]$. Extend $X, Y$ to $\mathcal{C}^{2}$ real vector fields in a neighbourhood of $\gamma\left(I_{\varepsilon}\right)$ on $\partial G$ which are complex tangent to $\partial G$ at each point. Let $v:[-\sigma, \sigma] \rightarrow W$ be a $\mathcal{C}^{2}$ curve which is transverse to the Levi leaves, with $v(0)=p$. Put $\tilde{H}(x+i y, t)=\mathcal{F}_{Y, y} \circ \mathcal{F}_{X, x} \circ v(t)$. For fixed $t$ the mapping $\tilde{\mathcal{H}}$ maps $R_{t}$ into the Levi-leaf through $v(t)$. The mapping may not be holomorphic in $z=x+i y$, but it is $\mathcal{C}^{1}$ close to the holomorphic map $z \rightarrow \mathcal{H}(z, 0)$.

${ }^{1}$ All further constructions depend on the point $p$, but we will skip the index $p$. 
There is a $\mathcal{C}^{1}$ family of reparametrizations $\varphi_{t}$ (each $\varphi_{t}$ being a map from a neighbourhood of $I_{\varepsilon}$ to a neighbourhood of $I_{\varepsilon}$ ) such that $\varphi_{\circ}=\mathrm{id}$ and

$$
\mathcal{H}(z, t)=\tilde{\mathcal{H}}\left(\varphi_{t}(z), t\right)
$$

is holomorphic for each $t$. Indeed, denote by $\tilde{\mathcal{H}}_{j}$ the coordinate functions of $\tilde{\mathcal{H}} . \varphi_{t}$ must satisfy the equations

$$
\bar{\partial}\left(\tilde{\mathcal{H}}_{j}\left(\varphi_{t}, t\right)\right)=\partial \tilde{\mathcal{H}}_{j}\left(\varphi_{t}, t\right) \bar{\partial} \varphi_{t}+\bar{\partial} \tilde{\mathcal{H}}_{j}\left(\varphi_{t}, t\right) \overline{\partial \varphi_{t}}=0, \quad j=1,2 .
$$

Each of the two equations (2.8) gives a well defined non-linear $\bar{\partial}$-operation for $\varphi_{t}$ on the set where the respective $\partial \tilde{\mathcal{H}}_{j}$ does not vanish. Since for fixed $t, \tilde{\mathcal{H}}(z, t)=$ $\left(\tilde{\mathcal{H}}_{1}(z, t), \tilde{\mathcal{H}}_{2}(z, t)\right)$ maps into a Levi-leaf, there is an analytic function $F_{t}$ with non-vanishing gradient such that

$$
F_{t}\left(\tilde{\mathcal{H}}_{1}(z, t), \tilde{\mathcal{H}}_{2}(z, t)\right)=0
$$

for $z$ in a neighbourhood of $I_{\varepsilon}$. Taking $\partial$ - and $\bar{\partial}$-derivatives of (2.9) and taking into account that $\tilde{\mathcal{H}}(\cdot, t)$ is $\mathcal{C}^{1}$ close to an analytic diffeomorphism we obtain that if $\partial_{j} F_{t} \neq 0$ then $\partial \tilde{\mathcal{H}}_{i} \neq 0$ for the other index $i$ and at the set where both derivatives $\partial_{1} F_{t}$ and $\partial_{2} F_{t}$ do not vanish, $\frac{\bar{\partial} \mathcal{H}_{1}}{\partial \mathcal{H}_{1}}=\frac{\bar{\partial} \mathcal{H}_{2}}{\partial \mathcal{H}_{2}}$. This gives a well-defined $\bar{\partial}$-equation for $\varphi_{t}$ in a neighbourhood of $I_{\varepsilon}$ which has a solution close to the identity. Moreover, the solution $\varphi_{t}$ depends $\mathcal{C}^{1}$ on $t$ because $\tilde{\mathcal{H}}$ is of class $\mathcal{C}^{2}$. The mapping $\mathcal{H}$ is constructed on the lower face $Q \cap\left\{\operatorname{Im} z_{2}=0\right\}$.

To define $\mathcal{H}$ on the whole $Q$ we denote for $z \in \partial G$ by $n(z)$ the unit inner normal to $\partial G$. This is a $\mathcal{C}^{2}$ vector field on $\partial G$. Approximate $n$ on the compact set $\gamma([-\varepsilon, 1])$ by an analytic vector field $N$ in a neighbourhood (in $\mathbb{C}^{2}$ ) of this set ([8]). Put

$$
\mathcal{H}(z, t+i s)=\mathcal{F}_{N, s}(\mathcal{H}(z, t)),
$$

$s \in[0, \sigma]$ for a small positive number $\sigma$.

Since $N$ is holomorphic in a neighbourhood of $\gamma\left(I_{\varepsilon}\right)$ and $\mathcal{H}(z, t)$ is holomorphic in $z$, the function $\mathcal{H}(z, t+i s)$ is holomorphic in $z$. The construction of $\mathcal{H}$ on $Q$ is completed.

Decreasing $\varepsilon>0$ and $\sigma>0$ if necessary we may assume that the "right" "lower" 2-face of $Q_{\varepsilon, \sigma}, Q_{\varepsilon, \sigma} \cap\left\{\operatorname{Re} z_{1}=1, \operatorname{Im} z_{2}=0\right\}$, is mapped into $\stackrel{\circ}{V} \backslash \overline{\Psi(G)}$. Denote the "right" face $Q_{\varepsilon, \sigma} \cap\left\{\operatorname{Re} z_{1}=1\right\}$ by $Q_{\varepsilon, \sigma}^{r}$.

The following lemma states that at points of $\mathcal{H}\left(Q_{\varepsilon, \sigma}^{r}\right)$ the function has the desired continuity properties. The proof uses the local Theorem I and a pluriharmonic measure estimate.

Lemma 2.6. The estimate

$$
\sup _{\substack{p^{\prime}, p^{\prime \prime} \in \mathcal{H}\left(Q_{\varepsilon, \sigma}^{r}\right) \\\left|p^{\prime}-p^{\prime \prime}\right|<\delta}}\left|f\left(p^{\prime}\right)-f\left(p^{\prime \prime}\right)\right| \leq C^{\prime} v(\delta)^{1-\tau^{\prime}} \mu(\delta)^{\tau^{\prime} \text { def }} \tilde{\mu}(\delta)
$$

holds for a constant $\tau^{\prime} \in(0,1)$ and a constant $C^{\prime}$. 
Proof. Let $p^{\prime}, p^{\prime \prime} \in G$ be two points in a sufficiently small neighbourhood of $\mathcal{H}\left(Q_{\varepsilon, \sigma}^{r}\right),\left|p^{\prime}-p^{\prime \prime}\right|<\delta$ for a small $\delta>0$. Suppose first that one of the points, say $p^{\prime}$, has distance to $\partial G$ not exceeding $\delta$. Then, since $\mathcal{H}\left(Q_{\varepsilon, \sigma}^{r} \cap\left\{\operatorname{Im} z_{2}=0\right\}\right) \subset \stackrel{\circ}{V}$, there is a point $p^{*} \in \stackrel{\circ}{V}$ such that $\left|p^{\prime}-p^{*}\right| \leq \delta$. By the local Theorem I,

$$
\left|f\left(p^{\prime}\right)-f\left(p^{*}\right)\right| \leq \text { const } \mu(\delta) .
$$

The same inequality with $\delta$ replaced by $2 \delta$ holds for $p^{\prime \prime}$. Triangle inequality and properties of $\mu$ yield

$$
\left|f\left(p^{\prime}\right)-f\left(p^{\prime \prime}\right)\right| \leq C^{\prime \prime} \mu(\delta)
$$

for this case.

Let now $p^{\prime}, p^{\prime \prime} \in G$ be contained in a neighbourhood $\mathcal{N}$ of $\mathcal{H}\left(Q_{\varepsilon, \sigma}^{r}\right), \mid p^{\prime}-$ $p^{\prime \prime} \mid<\delta$ and both have distance bigger than $\delta$ to the boundary $\partial G$. The function

$$
q \rightarrow f(q)-f\left(p^{\prime}-p^{\prime \prime}+q\right)
$$

is analytic in the intersection $\mathcal{N} \cap$ \{dist $(q, \partial G)>\delta\}$, satisfies (2.10) on its boundary part contained in $\{$ dist $(q, \partial G)=\delta\}$ and has supremum norm not exceeding $\nu(\delta)$. Pluriharmonic measure of the latter boundary part with respect to $\mathcal{N} \cap$ $\{\operatorname{dist}(q, \partial G)>\delta\}$ computed at points of $\mathcal{H}\left(Q_{\varepsilon, \sigma}^{r}\right) \cap\{\operatorname{dist}(q, \partial G)>\delta\}$ has an estimate from below by a positive constant $\tau^{\prime}$. (This can be seen by slicing with complex lines). Hence, on $\mathcal{H}\left(Q_{\varepsilon, \sigma}^{r}\right) \cap\{q \in G$ : dist $(q, \partial G)>\delta\}$ the absolute value of the function (2.11) does not exceed

$$
\left(C^{\prime \prime} \mu(\delta)\right)^{\tau^{\prime}} \nu(\delta)^{1-\tau^{\prime}}
$$

The value of the function (2.11) at the point $q=p^{\prime} \in \mathcal{H}\left(Q_{\varepsilon, \sigma}^{r}\right) \cap\{\operatorname{dist}(q, \partial G)>\delta\}$ equals $f\left(p^{\prime}\right)-f\left(p^{\prime \prime}\right)$. This proves the required inequality in the second case. Lemma 2.6 is proved.

End of proof of Lemma 2.4. Let $\varepsilon^{\prime} \in(0, \varepsilon)$ and consider the rectangular box $Q_{\varepsilon^{\prime}, \sigma}$ obtained from $Q_{\varepsilon, \delta}$ by shrinking in the $z_{1}$-direction. We will prove that estimate (2.2) of the lemma holds on the set $\mathcal{H}\left(Q_{\varepsilon^{\prime}, \sigma}^{0}\right)$, where $Q_{\varepsilon^{\prime}, \sigma}^{0}$ is the intersection of the interior of $Q_{\varepsilon^{\prime}, \sigma}$ with $\left\{\left|\operatorname{Re} z_{1}\right|<\varepsilon^{\prime}\right\}$ in $\mathbb{C}^{2}$,

$$
Q_{\varepsilon^{\prime}, \sigma}^{0} \stackrel{\text { def }}{=}\left\{\left(z_{1}, z_{2}\right): z_{1} \in\left(-\varepsilon^{\prime}, \varepsilon^{\prime}\right)+i\left(\varepsilon^{\prime}, \varepsilon^{\prime}\right), z_{2} \in(-\sigma, \sigma)+i(0, \sigma)\right\} .
$$

The set $\mathcal{H}\left(Q_{\varepsilon^{\prime}, \sigma}^{0}\right)$ is contained in $G$ and contains the intersection of $G$ with a ball around $p$. (Recall, that $\mathcal{H}(0,0)=p$.)

The diffeomorphism $\mathcal{H}$ and its inverse $\mathcal{H}^{-1}$ preserve distance up to a multiplicative constant, i.e.

$$
\begin{aligned}
\left|\mathcal{H}^{-1}\left(p^{\prime}\right)-\mathcal{H}^{-1}\left(p^{\prime \prime}\right)\right| & \leq c^{\prime}\left|p^{\prime}-p^{\prime \prime}\right|, \quad p^{\prime}, p^{\prime \prime} \in \mathcal{H}\left(Q_{\varepsilon, \sigma}\right), \\
\left|\mathcal{H}\left(z^{\prime}\right)-\mathcal{H}\left(z^{\prime \prime}\right)\right| & \leq c^{\prime \prime}\left|z^{\prime}-z^{\prime \prime}\right|, \quad z^{\prime}, z^{\prime \prime} \in Q_{\varepsilon, \sigma} .
\end{aligned}
$$


Let $p^{\prime}, p^{\prime \prime} \in \mathcal{H}\left(Q_{\varepsilon^{\prime}, \sigma}^{0}\right),\left|p^{\prime}-p^{\prime \prime}\right|<\delta$ with $\delta>0$. Write $\mathcal{H}^{-1}\left(p^{\prime}\right)=\left(z_{1}^{\prime}, z_{2}^{\prime}\right) \in$ $Q_{\varepsilon^{\prime}, \sigma}, \mathcal{H}^{-1}\left(p^{\prime \prime}\right)=\left(z_{1}^{\prime \prime}, z_{2}^{\prime \prime}\right) \in Q_{\varepsilon^{\prime}, \sigma}$ and consider the function

$$
z \rightarrow(f \circ \mathcal{H})\left(z, z_{2}^{\prime}\right)-(f \circ \mathcal{H})\left(z+\left(z_{1}^{\prime \prime}-z_{1}^{\prime}\right), z_{2}^{\prime \prime}\right) .
$$

If $\delta>0$ is small, this function is in $A(R)$ for the rectangle $R=[-\varepsilon, 1]+i[-\varepsilon, \varepsilon]$. (Note that $\operatorname{Im} z_{2}^{\prime}>0, \operatorname{Im} z_{2}^{\prime \prime}>0$ ). The value of the function (2.12) at $z_{1}^{\prime}$ equals

$$
(f \circ \mathcal{H})\left(z_{1}^{\prime}, z_{2}^{\prime}\right)-(f \circ \mathcal{H})\left(z_{1}^{\prime \prime}, z_{2}^{\prime \prime}\right)=f\left(p^{\prime}\right)-f\left(p^{\prime \prime}\right) .
$$

The supremum norm of the function (2.12) does not exceed $v\left(c^{\prime} c^{\prime \prime} \delta\right)$. On the right side $\{1\} \times i[-\varepsilon, \varepsilon]$ of the rectangle $R$ its absolute value does not exceed $\tilde{\mu}\left(c^{\prime} c^{\prime \prime} \delta\right)$. Therefore on the relatively compact part $\left\{\left(-\varepsilon^{\prime}, \varepsilon^{\prime}\right)+i\left(-\varepsilon^{\prime}, \varepsilon^{\prime}\right)\right\}$ of $R$, in particular at the point $z_{1}^{\prime}$, its absolute value does not exceed

$$
C_{p} v(\delta)^{1-\tau_{p}} \mu(\delta)^{\tau_{p}}
$$

for suitable constants $\tau_{p} \in(0,1]$ and $C_{p}>0$. Here we used property (1.3) of $\mu$ and $v$, the fact that $v \geq \mu$, the definition of $\tilde{\mu}$ in Lemma 2.6 and harmonic measure estimate on $R$.

Lemma 2.4, and hence theorems 1.3 and 2.1, are proved.

We do not know whether in theorems 1.3 and 2.1 one needs to require that the function $f$ is bounded in $G$.

Under some additional hypothesis this condition can be removed. The following lemma describes sufficient conditions in the case of pseudoconvex domains.

Lemma 2.7. Let $G \subset \mathbb{C}^{2}$ be a bounded pseudoconvex domain with $\mathcal{C}^{2}$ boundary. Suppose each set of an exhausting sequence of compact subsets of $\partial G \backslash \overline{\Psi_{+}(G)}$ has a Stein neighbourhood basis. Then any analytic function in $G$ that extends continuously to a neighbourhood of $\overline{\Psi_{+}(G)}$ in $\partial G$ is bounded in $G$.

Proof. Let $V$ be a neighbourhood of $\overline{\Psi_{+}(G)}$ in $\partial G$ and $f$ a continuous function on $G \cup V$ that is holomorphic in $G$. Let $\stackrel{\circ}{V}$ be open in $\partial G$ and $\overline{\Psi_{+}(G)} \subset \stackrel{\circ}{V} \Subset V$. There is a compact subset $K \subset G \cup V$ which contains a one-sided neighbourhood of each point of $\stackrel{\circ}{V}$ (i.e. the intersection of $G$ with a small ball around the point). The function $f$ is bounded on $K$.

The set $\partial G \backslash \stackrel{\circ}{V}$ is a compact subset of $\partial G \backslash \overline{\Psi_{+}(G)}$. It is contained in a compact set $\kappa \subset \partial G \backslash \overline{\Psi_{+}(G)}$ which has a Stein neighbourhood basis. Since any pseudoconvex domain can be exhausted by smoothly bounded strictly pseudoconvex domains, there exists a neighbourhood basis $U_{n}$ of $\kappa$ consisting of smoothly bounded strictly pseudoconvex domains. Consider $G \backslash U_{n}$ and smoothen its boundary part which is contained in a small neighbourhood of $\partial U_{n} \cap \partial G \subset \stackrel{\circ}{V}$. We may assume that the changed boundary part is contained in $K$ and the thus obtained domain $G_{n}$ has $\mathcal{C}^{2}$ boundary. 
The function $f$ is continuous on $\bar{G}_{n} \subset G \cup V$. The part of the boundary $\partial G_{n}$ which is outside $K$ is strictly pseudoconcave hence does not meet $\overline{\Psi_{+}\left(G_{n}\right)}$. By Theorem III

$$
\max _{\bar{G}_{n}}|f| \leq \max _{K}|f| .
$$

This is true for all $n$ and the $G_{n}$ exhaust $G$. The lemma is proved.

Necessary and sufficient conditions for the existence of a Stein neighbourhood basis of a Levi-flat hypersurface are not known. See [3] for an account.

\section{References}

[1] R. F. BASEner, Peak points, barriers and pseudoconvex boundary points, Proc. Amer. Math. Soc. 65 (1977), 89-92.

[2] H. J. BREMERMANN, On a generalized Dirichlet problem for plurisubharmonic functions and pseudo-convex domains. Characterization of Šilov boundaries, Trans. Amer. Math. Soc. 91 (1959), 246-276.

[3] F. Forstneric, and C. LAURENT-ThiÉBAUt, Stein compacts in Levi-flat hypersurfaces, Trans. Amer. Math. Soc. 360 (2008), 307-329.

[4] T. W. Gamelin, "Uniform Algebras”, Prentice-Hall Inc., Englewood Cliffs, N. J., 1969.

[5] I. Glicksberg, Boundary continuity of some holomorphic functions, Pacific J. Math. 80 (1979), 425-434.

[6] S. J. GREENFIELD, Cauchy-Riemann equations in several variables, Ann. Scuola Norm. Sup. Pisa Cl. Sci. 22 (1968), 275-314.

[7] G. H. Hardy and J. E. LitTlewood, Some properties of fractional integrals. II, Math. Z. 34 (1932), 403-439.

[8] F. R. HARVEY and R. O. Wells, Holomorphic approximation on totally real submanifolds of a complex manifold, Bull. Amer. Math. Soc. 77 (1971), 824-828.

[9] B. JÖRICKE, The relation between the solid modulus of continuity and the modulus of continuity along a Shilov boundary for analytic functions of several variables, Mat. Sb. (N.S.) 122 (164) (1983), 511-526.

[10] B. JÖRICKE, The modulus of continuity of analytic functions in a domain and on its Shilov boundary, In: "Problems in the Theory of Functions of Several Complex Variables and in Infinite-Dimensional Complex Analysis", Lecture Notes in Math., Vol. 1039, Springer, 1983, Collected and prepared by Christer O. Kiselman, 472-473.

[11] B. JÖRICKE, Some remarks concerning holomorphically convex hulls and envelopes of holomorphy, Math. Z. 218 (1995), 143-157.

[12] P. Pflug, Über polynomiale Funktionen auf Holomorphiegebieten, Math. Z. 139 (1974), 133-139.

[13] H. Rossi, Holomorphically convex sets in several complex variables, Ann. of Math. 74 (1961), 470-493.

[14] L. A. Rubel, A. L. Shields and B. A. TAYlor, Mergelyan sets and the modulus of continuity of analytic functions, J. Approx. Theory 15 (1975), 23-40.

[15] W. E. SEWELL, "Degree of Approximation by Polynomials in the Complex Domain", Annals of Mathematical Studies, n. 9, Princeton University Press, Princeton, N. J., 1942. 
[16] J.-M. TRÉPREAU, Sur le prolongement holomorphe des fonctions $C$-R défines sur une hypersurface réelle de classe $C^{2}$ dans $\mathbf{C}^{n}$, Invent. Math. $\mathbf{8 3}$ (1986), 583-592.

[17] A. E. Tumanov, Extension of CR-functions into a wedge, Mat. Sb. 181 (1990), 951-964.

Department of Mathematics Stockholm University SE-106 91 Stockholm, Sweden salla@math.su.se

Max-Planck-Institut für Mathematik

P.O. Box: 7280

53072 Bonn, Germany and

Department of Mathematics Stockholm University SE-106 91 Stockholm, Sweden joericke@math.su.se 